\title{
AOK-Bundesverbandsspitze wieder komplett
}

Der Volkswirt Jens Martin Hoyer komplettiert den Vorstand des AOK-Bundeverbandes als stellvertretender Vorsitzender. Der Aufsichtsrat des Verbandes wählte den 48-Jährigen zum Stellvertreter von Vorstandschef Martin Litsch. Im Geschäftsführenden Vorstand wird er sich vor allem um die Bereiche Fi- nanzen und Controlling kümmern. Bereits von 1999 bis 2008 war Hoyer im AOK-Bundesverband tätig. Danach wechselte er zur Techniker Krankenkasse nach Hamburg, wo er die Bereiche Unternehmensentwicklung und Controlling leitete.

\section{DAK-Gesundheit}

\section{Ex-Gesundheitsminister des Saarlands wird Krankenkassenchef}

Der ehemalige saarländische Gesundheitsminister Andreas Storm (CDU) wird Chef der DAK-Gesundheit. Das entschied der Verwaltungsrat der drittgrößten deutschen Krankenkasse auf seiner Sitzung Ende März. Der Plan des Verwaltungsrates sieht vor, dass der Weg an die Spitze der Kasse in zwei Phasen verläuft. Ab Juli soll Storm in den Vorstand aufrücken und den aktuellen Vorstandsvize Claus Moldenhauer beerben. Zum Jahresbeginn 2017 wird Storm dann den Posten des derzeitigen
Vorsitzenden von Herbert Rebscher übernehmen, der in den Ruhestand geht.

In der Gesundheitspolitik ist Storm bekannt. Zwischen 1994 und 2009 war er Mitglied des Deutschen Bundestages. In seiner Arbeit als Abgeordneter zeichnete er sich durch seine Expertise in der Renten- und Gesundheitspolitik aus. Von Mai 2012 bis November 2014 war er Minister für Soziales, Gesundheit, Frauen und Familie im Saarland.

cas

\section{Herzlichen Glückwunsch}

\section{Dietmar Oesterreich wird 60}

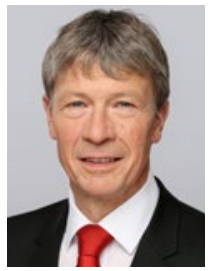

Am 28. Mai 2016 feiert Prof. Dr. Dietmar Oesterreich seinen 60. Geburtstag. In den 26 Jahren als Präsident der Zahnärztekammer Mecklenburg-Vorpommern hat er die zahnärztliche Standespolitik mitgeprägt. Nach seinem Zahnmedizinstudium in Rostock war er bis 1990 in der Poliklinik für Stomatologie in Malchin in der Mecklenburgischen Schweiz tätig. Seit 1991 ist er in der Fritz-Reuter-Stadt Stavenhagen niedergelassen. In der Zeit der Wende hat er sich sofort standespolitisch engagiert und wurde 1990 auch erster Präsident der Zahnärztekammer Mecklenburg-Vorpommern. In den Folgejahren hat Oesterreich sich beispielhaft für die notwendige Umstrukturierung des zahnärztlichen Gesundheitswesens in Mecklenburg-Vorpommern und die Fortentwicklung der zahnärztlichen Selbstverwaltung eingesetzt. Insbesondere für diese Verdienste wurde er im Jahr 2004 mit dem Bundesverdienstkreuz am Bande des Verdienstordens der Bundesrepublik Deutschland ausgezeichnet.

Mitglied im Freien Verband ist Oesterreich seit Anfang 1991. Seit November 2000 ist er auch Vizepräsident der Bundeszahnärztekammer (BZÄK). Sehr am Herzen liegen ihm seit jeher die Kinder- und Jugendzahnheilkunde und die Prävention in jedem Lebensalter. Als Vorsitzender des Ausschusses „Präventive Zahnheilkunde" der BZÄK war er wesentlich an der Neubeschreibung der präventionsorientierten Zahnheilkunde beteiligt. Akzente konnte er auch als Referent der BZÄK für „Patientenberatung “ und als Referent für „Öffentlichkeitsarbeit" setzen. Weitere Schwerpunkte seiner Tätigkeit findet man in den Referaten „Wissenschaft und Forschung in der Zahnmedizin“

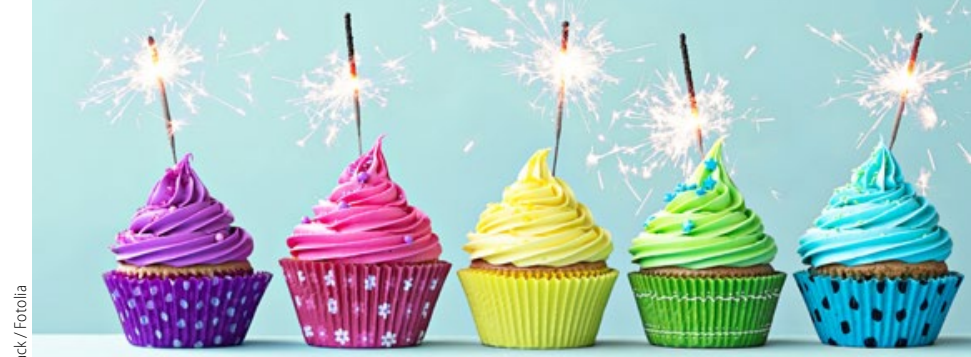

und „Förderung des beruflichen Nachwuchses“, als alternierender Vorsitzender der DAJ, als Mitglied des Vorstandsausschusses des Instituts der Deutschen Zahnärzte oder bei der Initiative proDente. Oesterreich widmet sich insbesondere der Versorgungsforschung. In seinen Publikationen finden wir aber auch einen Leitfaden der präventionsorientierten Zahnheilkunde unter den besonderen Aspekten des Alterns oder Themenbearbeitungen zur Psychosomatik in der Zahnheilkunde.

Im Jahr 2011 erhielt Oesterreich eine Honorarprofessur für Orale Prävention und Versorgungsforschung an der Klinik und Poliklinik für Mund-Kiefer-Gesichtschirurgie/Plastische Operationen der Universität Greifswald.

$\mathrm{Zu}$ seinem Geburtstag beglückwünschen wir ihn und hoffen, dass er noch viele Jahre an der zahnärztlichen Standespolitik Freude findet. Dr. Peter Bührens 